\title{
Philonsorbonne
}

15 | 2021

Année 2020-2021

\section{L'usage féminin du savoir : plaisir de l'étude et émancipation chez Poulain de la Barre et Émilie du Châtelet}

\section{Clément RAYMOND}

\section{(2) OpenEdition \\ Journals}

Édition électronique

URL : https://journals.openedition.org/philonsorbonne/1836

DOI : 10.4000/philonsorbonne.1836

ISSN : 2270-7336

Éditeur

Publications de la Sorbonne

Édition imprimée

Date de publication : 1 janvier 2021

Pagination : 95-108

ISSN : 1255-183X

Référence électronique

Clément RAYMOND, «L'usage féminin du savoir : plaisir de l'étude et émancipation chez Poulain de la Barre et Émilie du Châtelet », Philonsorbonne [En ligne], 15 | 2021, mis en ligne le 03 février 2021, consulté le 08 juin 2021. URL : http://journals.openedition.org/philonsorbonne/1836 ; DOI : https:// doi.org/10.4000/philonsorbonne.1836

(c) Tous droits réservés 


\title{
L'usage féminin du savoir : plaisir de l'étude et émancipation chez Poulain de la Barre et Émilie du Châtelet
}

\author{
Clément RAYMOND
}

\section{Introduction}

(a) Ce que les femmes peuvent savoir : enjeux épistémiques et politiques

Déterminer ce que les femmes sont capables de connaître et ce qu'il est bon qu'elles connaissent: voilà un enjeu qui traverse les textes qui composent la Querelle des femmes. Il relève évidemment de la théorie de la connaissance : la puissance rationnelle des femmes est-elle de même nature et de même étendue que celle des hommes ? Certains caractères réputés féminins (la sensibilité, un certain tempérament, des vices ou vertus propres au sexe) sont-ils susceptibles d'aider ou de contrarier les études? Mais cet enjeu est également de philosophie politique, tant sont nombreuses les connexions entre les connaissances dont les femmes sont réputées capables et les positions sociales qui sont ou doivent (supposément) être les leurs. On pourra, à l'instar d'un Fénelon, partir d'une fonction propre à la femme dans la société (« une maison à régler, un mari à rendre heureux, des enfants à bien élever $»^{1}$ ) pour en déduire le type de connaissances qu'elle doit chercher à acquérir : "La science des femmes, comme celle des hommes, doit se borner à s'instruire par rapport à leurs fonctions $»^{2}$. On pourra au contraire partir d'un défaut d'aptitude au savoir supposé naturel chez les femmes pour leur interdire certains champs disciplinaires et donc certaines positions

1. Fénelon, De l'éducation des filles, ch. I : « De l'importance de l'éducation des filles », dans Fénelon, Euvres, J. le Brun (éd.), Paris, Gallimard, 1983, t. I, p. 93.

2. Ibid., ch. IX, p. 154. 
ou charges sociales. C'est ce que fera l'initiateur de la «querelle des alphabets », Jacques Olivier, affirmant que la femme « est un sexe si fragile et si muable, [qu'il] semble que la nature l'ait seulement forgée pour la perpétuité de l'espèce humaine ${ }^{3}$, ou encore qu'elle ne devrait avoir aucune charge publique, "manquant par trop de jugement et de sagesse pour la conduite du bien et des affaires d'un public $»^{4}$.

Si le lien entre la misogynie dans la théorie de la connaissance et la misogynie dans la théorie politique et sociale est déjà étudié, c'est moins le cas pour la "philogynie ». Nous proposons ainsi d'aborder la thèse de l'égalité épistémique selon le sexe, c'est-à-dire de l'égale capacité de savoir des hommes et des femmes ${ }^{5}$. Nous voulons l'étudier dans les relations qu'elle peut entretenir avec les affirmations 1) de la nécessité de l'éducation des dames et 2) de l'identité de l'éducation des femmes et des hommes. Autrement dit, nous allons nous intéresser moins à la démonstration de l'égalité des deux sexes pour elle-même que dans la relation qu'elle entretient avec l'affirmation de l'importance de l'éducation féminine. De l'égalité des esprits à la nécessité de l'éducation de tous les esprits, ou à l'identité de l'éducation de tous les esprits, la conséquence n'est pas directe. Pour le mesurer, nous rappellerons d'abord la thèse de l'égalité épistémique selon le sexe telle que l'a formulée le cartésien Poulain de la Barre ${ }^{6}$.

\section{(b) La thèse de Poulain de la Barre : l'égale capacité des hommes et des femmes pour la connaissance}

L'objectif de Poulain, dans sa démonstration de l'égalité des deux sexes, est d'affirmer «[q]ue les femmes considérées selon les principes de la saine Philosophie, sont autant capables que les hommes de toutes sortes de connaissances » $(E S$, II, p. 99). Il le démontre en deux temps : d'abord, en établissant que l'esprit n'a point de sexe, c'est-à-dire que la capacité de bien juger est indépendante de la différence sexuelle; ensuite, en montrant que le corps, en relation avec lequel l'esprit pense le plus souvent, ne diffère naturellement en rien entre les hommes et les femmes, si ce n'est par les organes sexuels.

3. Jacques Olivier, Alphabet de l'imperfection et malice des femmes..., Paris, chez Jean PetitPas, 1617, «Bestiale barathrum - Abîme de bêtise », p. 37.

4. Ibid., p. 39.

5. L'expression est reprise à Louise Marcil-Lacoste dans son article : « L'héritage cartésien : l'égalité épistémique », Philosophiques, 15 (1), (1988), p. 77-94. Il ne s'agit pas pour nous de mentionner la seule capacité des esprits (comme choses pensantes), mais l'ensemble des éléments qui concourent à la connaissance. L'égalité épistémique, appliquée à la différence des sexes, signifie non seulement que les esprits des hommes et des femmes sont de même nature et aptes aux mêmes savoirs, mais aussi que l'on ne trouvera dans les secondes aucun défaut naturel particulier qui contreviendrait aux études.

6. Nous citerons ses textes d'après: Poulain de la Barre, De l'égalité des deux sexes, De l'éducation des dames, De l'excellence des hommes, M.-F. Pellegrin (éd.), Paris, Vrin, 2017. Les titres des trois traités sont abrégés, successivement, $E S, E D$ et $E H$. 
Si l'esprit, c'est-à-dire la substance pensante, «n'a point de sexe» (ES, II, p. 100), c'est parce que le sexe « ne regarde que le Corps : n'y ayant proprement que cette partie qui serve à la production des hommes » $(E S$, II, p. 99). La distinction des sexes regarde la propagation ${ }^{7}$ de l'espèce humaine, pour laquelle le corps est le seul acteur. Relativement à la faculté de penser en général et de connaître en particulier, être homme ou femme semble n'impliquer aucune différence : l'esprit étant de même nature en tous les êtres humains, il est caractérisé en chacun par les mêmes capacités. Mais évidemment, quoique la pensée ressortisse à l'esprit seul, ce dernier est uni à un corps duquel il reçoit des informations à partir desquelles il pense. Il est donc primordial de montrer que le corps féminin n'est pas naturellement une plus grande source d'erreur et d'embarras pour l'esprit que le corps masculin. L'argumentation poulainienne consiste à mobiliser la physiologie de Descartes pour contrecarrer la théorie humorale de Galien ${ }^{8}$ et réduire la différence sexuelle, déjà cantonnée au niveau du corps, à la seule possession d'organes sexuels différenciés'. Poulain souligne notamment l'absence de différence entre les cerveaux féminins et masculins, de sorte que, quel que soit le sexe, «les impressions des sens s'y [dans le cerveau] reçoivent, et s'y rassemblent de même façon et ne s'y conservent point autrement pour l'Imagination et pour la Mémoire. Les femmes entendent comme nous, par les oreilles; elles voient par les yeux, et elles goûtent avec la langue » (ES, II, p. 101).

La démonstration poulainienne est ici achevée: la nature de l'esprit est identique dans les hommes et les femmes et l'étude ne rencontre pas plus d'obstacles dans les secondes que dans les premiers; il est ainsi capable, quel que soit le sexe du corps auquel il est uni, des mêmes connaissances. Pour autant, de cette thèse de l'égalité épistémique, il ne faut pas conclure directement à la nécessité de l'éducation identique de tous les esprits. Stasimaque, représentant de Poulain dans De l'éducation des dames, dit plutôt :

Ce que je voudrais, ce serait seulement que l'on instruisît les femmes ${ }^{10}$, avec autant de soin que les hommes; parce que cela serait très utile, et ne nuirait à personne. $(E D, \mathrm{I}, \mathrm{p} .173, \mathrm{~ns}$.)

\footnotetext{
7. Terme de Marie de Gournay, dans un contexte similaire (Égalité des hommes et des femmes, dans ses Euvres complètes, J.-C. Arnould (éd.), Paris, Honoré Champion, 2002, t. 1, p. 978).

8. Voir par exemple en EH, I, p. 345-346.

9. Pour une analyse étendue de l'argumentation poulainienne, on se reportera à : M.-F. Pellegrin, Pensées du corps et différences des sexes à l'époque moderne. Descartes, Cureau de la Chambre, Poulain de la Barre et Malebranche, Lyon, ENS Éditions, 2020 (en particulier au ch. 8 : « La vraie femme », p. 349-400).

10. Pour une présentation complète de la théorie poulainienne de l'instruction des femmes, nous renvoyons à M. Corona Corrias, Alle origini del femminismo moderno. Il pensiero politico di Poullain de la Barre, Milan, Franco Angeli, 1996 (en particulier au ch. 5 : «II problema dell'istruzione $»)$.
} 
Entre l'égalité et la nécessité de l'éducation des femmes, Poulain de la Barre mobilise deux types d'arguments différents, qui reposent chacun sur un concept particulier: le "droit sur la vérité », d'une part, le «bon usage » de l'étude, d'autre part. Selon le premier, les femmes doivent recevoir la même éducation que les hommes, car le droit sur la vérité est le même pour tout être humain. Selon le second, les femmes sont aussi susceptibles que les hommes d'un bon usage de l'étude, qui sera utile à chacune d'entre elles individuellement comme à la société en général. Ce sont ces arguments, et les deux concepts nodaux sur lesquels il se construisent, que nous étudierons, en les mettant en relation avec d'autres positions féministes ${ }^{11}$ des XVII ${ }^{\mathrm{e}}$ et XVIII ${ }^{\mathrm{e}}$ siècles.

\section{Le « droit sur la vérité »}

Pour passer de l'égalité des deux sexes à la nécessité de l'éducation, Poulain de la Barre mobilise d'abord un concept intermédiaire, celui de « droit sur la vérité » ou sur « tout ce qui est du bon sens » :

En effet nous avons tous, hommes et femmes, le même droit sur la vérité, puisque l'esprit est en tous également capable de la connaître, et que nous sommes tous frappés de la même façon, par les objets qui font impression sur le corps. Ce droit que la nature nous donne à tous sur les mêmes connaissances, naît de ce que nous en avons tous autant de besoin les uns que les autres. Il n'y a personne qui ne cherche à être heureux, et c'est à quoi tendent toutes nos actions ; et pas un ne le peut être solidement que par des connaissances claires, et distinctes [...]. (ES, II, p. 111)

Chacun a droit sur tout ce qui est du bon sens : le Ressort de la raison n'a point de borne; elle a dans tous les hommes une égale juridiction. Nous naissons tous juges des choses qui nous touchent; et si nous ne pouvons pas tous en disposer avec un pouvoir égal, nous pouvons au moins les connaître tout également. Et comme tous les hommes jouissent de l'usage de la lumière et de l'air, sans que cette communication soit préjudiciable à personne, tous peuvent aussi posséder la vérité sans se nuire les uns aux autres. Et même plus elle est connue, plus elle parait belle et lumineuse : plus il y a de personnes qui la cherchent, et plus tôt on la découvre : si les deux Sexes y avaient travaillé également, on l'aurait plus tôt trouvée. De sorte que la vérité et la science sont des biens imprescriptibles : et ceux qui en ont été privés y peuvent rentrer sans faire tort à ceux qui en sont déjà les maîtres. Il ne peut donc y avoir que ceux qui veulent dominer sur les esprits par la créance, qui aient sujet d'appréhender ce retour, dans la crainte que si les sciences devenaient si communes, la gloire ne le devint aussi, et que celle où ils aspirent, ne se diminuât par le partage. (ES, II, p. 115-116)

11. On appelle féministe un discours qui a pour objet l'abrogation de la sujétion sociale des femmes, l'amélioration de leurs conditions d'existence et l'extension de leurs droits. 
Dans les deux textes, le droit égal, quel que soit le sexe, mais généralement pour tout individu, sur la vérité ou sur tout ce qui est du bon sens, est inféré comme conclusion de l'égale capacité de connaître des esprits. L'omniprésence du vocabulaire du droit civil ${ }^{12}$ fait à la fois la teneur singulière du texte et le cour de son argumentation : à la suite de l'Apologie de la science des dames, publiées par l'anonyme Cléante en 1662 à Lyon ${ }^{13}$, Poulain de la Barre théorise ici la différence entre les biens de l'esprit, d'un côté, et les biens du corps, de l'autre. Il y a une spécificité de la science et de la vérité par rapport aux objets de jouissance corporelle qui tient à ce que tous les êtres humains peuvent les posséder sans se nuire les uns aux autres. C'est ce que l'économie contemporaine, à propos des biens publics et communs, appelle la non-rivalité d'un bien : le fait que sa consommation, sa possession ou son usage par un individu n'entraîne pas une diminution de la quantité de ce bien pour les autres ${ }^{14}$. On y peut ajouter une autre caractéristique que l'on trouve déjà dans l'Apologie de la science des dames: alors que "la possession [des biens du corps] ne peut jamais être sans exclusion ${ }^{15}$, il est impossible d'interdire la possession des biens de l'esprit, de la science et de la vérité. Pour reprendre une nouvelle fois le vocabulaire de l'économie contemporaine, science et vérité sont des biens non excluables ${ }^{16}$. Poulain de la Barre affirme, quant à lui, que ce sont des biens imprescriptibles, c'est-à-dire dont on ne peut revendiquer la propriété même si l'on en a acquis la possession ${ }^{17}$.

Quel est donc le type de droit que l'on peut revendiquer sur la vérité, ou sur tout ce qui est du bon sens? C'est d'abord un droit qui admet pour condition de possibilité l'égalité épistémique - sans elle, en effet, il resterait lettre morte. Il est ensuite légitimé par l'universalité du besoin de connaissances vraies : Poulain de la Barre - nous y reviendrons -, établit une identité entre le bonheur, recherché par tout être humain, et la connaissance vraie. On comprend par là qu'il s'agit d'un droit naturel ${ }^{18}$, et non d'une loi

12. Droit civil que Poulain de la Barre définit lui-même ainsi : «l'application de [l'autorité des Magistrats et des Officiers], dans les Lois, les Ordonnances, et les Règlements, pour la conduite de ceux qui y sont soumis, tant pour le rapport des personnes, selon les diverses conditions, que pour la possession et pour l'usage des biens » (ES, II, p. 109).

13. Cléante, Apologie de la science des dames, chez Benoist Coral, Lyon, 1662. Voir en particulier les p. 84-85. Il y est question «des biens de l'âme", aussi appelés «biens de l'esprit », et de « ceux de la terre », aussi appelés « biens grossiers ».

14. P. A. Samuelson, «The Pure Theory of Public Expenditure », The Review of Economics and Statistics, 36 (4), (1954), p. 387-389.

15. Apologie de la science des dames, op. cit., p. 84.

16. E. et V. Ostrom, «Public Goods and Public Choices », in E.S. Savas (éd.), Alternatives for Delivering Public Services: Toward Improved Performance, Boulder, Westview Press, p. 7-49.

17. Voir : ES, II, p. 115.

18. «Pour moi je ne vois rien que la nature ne leur [aux femmes] ait permis comme à nous [les hommes], leur ayant donné le même droit de faire tout ce qu'elles jugeront à propos pour la perfection de leur esprit et pour la conservation et le soulagement du corps ». ( $E H$, II, p. 382). 
positive : il se distingue cependant de l'égale capacité naturelle des esprits à la connaissance et désigne plutôt la liberté d'exercer cette capacité ${ }^{19}$; attaché à l'essence de l'être humain, il ne saurait dépendre du particularisme d'un droit positif. Enfin, pour terminer de clarifier le type de droit dont il est question, il faut revenir sur la notion de bien imprescriptible, qui recouvre une question juridique précise, au fondement du droit des biens. Nous avons utilisé à cette fin un Dictionnaire civil et canonique de la seconde moitié $\mathrm{du} \mathrm{XVII}^{\mathrm{e}}$ siècle $^{20}$. La possession est «la détention d'une chose $»^{21}$ et se distingue de la propriété ou "domaine d'une chose $»^{22}$, qui regroupe l'ensemble des pouvoirs (usus, fructus, abusus) que l'on est en droit d'exercer sur la chose. La prescription correspond à «l'acquisition du domaine d'une chose qu'on a possédée sans interruption $»^{23}$ pendant un certain temps, qui est défini par la loi, qui diffère selon le type de biens. Dire de la vérité et de la science qu'elles sont des biens imprescriptibles revient ainsi à affirmer que leur possession, quelque longue qu'elle soit, n'autorise pas pour autant à s'en réclamer propriétaire. Le droit sur la vérité n'est donc pas un droit de propriété mais un droit à la possession.

L'introduction de concepts relevant du droit civil à propos de la science et de la vérité, et le fait de les subsumer avec tous les objets de jouissance corporelle sous le concept de «bien» ne vont évidemment pas sans difficulté. D'abord, en raison de sa nature spirituelle et intérieure, on ne possède évidemment pas la science de la même manière que des biens meubles ou immeubles. Ensuite, le vocabulaire du droit des biens, et notamment les caractéristiques du droit de propriété, ne conviennent pas à ces biens non excluables et non rivaux que sont la vérité et la science : quel sens, par exemple, reste-t-il de l'abusus à propos de la vérité ? D'où le paradoxe auquel nous avons été réduits, en parlant de «droit de possession ». On voit bien ainsi que le rapport d'un esprit à la vérité excède de toute part ce que le droit nous dit de la possession de biens corporels.

Dès lors, pourquoi Poulain de la Barre a-t-il choisi de mobiliser des concepts relevant du droit civil pour penser la relation entre l'égalité épistémique et la nécessaire éducation des dames ? Il s'agit d'abord de répondre à un argument répandu qui exprime une crainte des hommes : eux qui avaient le monopole du savoir seraient mis en danger par une plus grande

19. Dans la citation donnée dans la note précédente, c'est la notion de permission qui différencie la capacité naturelle de l'esprit du droit naturel de l'exercer.

20. $\mathrm{Me}^{* * * * *}$ avocat au Parlement, Dictionnaire civil et canonique, contenant les étimologie, définitions, divisions et principes du droit français conféré avec le droit romain, et de la pratique accomodée aux nouvelles ordonnances, Paris, chez Augustin Besoigne et Jérôme Bobin, 1687. Dans les citations, nous avons conservé la ponctuation et les majuscules, mais modernisé l'orthographe. Que la publication du dictionnaire soit postérieure aux œuvres de Poulain n'enlève en rien sa pertinence pour notre enquête : il repose sur un examen du droit romain et des ordonnances et coutumes qui constituent le droit français depuis le $\mathrm{XVI}^{\mathrm{e}}$ siècle.

21. Ibid., art. « Possession », p. 658.

22. Ibid., art. « Domaine », p. 280.

23. Ibid., art. «Prescription », p. 667. 
accessibilité de la connaissance aux femmes ${ }^{24}$. Plus profondément, il s'agit de l'idée qu'en matière de vérité et de science, il existerait des intérêts divergents, ou des conflits de propriété relativement au savoir. Poulain s'attache donc à montrer au contraire que la question de la propriété ne se pose pas vis-à-vis de la vérité stricto sensu, mais vis-à-vis de la position de pouvoir (charge et autorité) de « ceux qui veulent dominer sur les esprits par la créance », tant dans la société en général que dans les institutions du savoir en particulier - les maîtres aristotéliciens. Ensuite, et peut-être surtout, Poulain de la Barre témoigne, par l'introduction des concepts de droit civil, de sa conscience d'un hiatus entre l'égale capacité des esprits et la reconnaissance du droit d'exercer cette capacité. L'histoire des sociétés humaines est celle de l'exclusion progressive des femmes en dehors des sphères du $\operatorname{savoir}^{25}$ : par la coutume, les règles de droit et les règlements des institutions, la capacité de savoir des femmes a été entravée, interdite d'exercice. C'est contre ce mouvement historique et les droits positifs des États que Poulain de la Barre prend le parti de réaffirmer l'existence d'un droit naturel des femmes et de tous les êtres humains à la connaissance ${ }^{26}$.

\section{Le bon usage du savoir}

Nous rappelions plus haut que pour Stasimaque-Poulain, l'instruction des femmes est nécessaire non seulement parce qu'elle «ne nuirait à personne », mais aussi parce qu'elle «serait très utile ». Le concept de droit sur la vérité et les caractéristiques propres aux biens de l'esprit par rapport aux biens du corps rendent raison de ce que l'éducation des dames n'entraînerait aucune nuisance - les deux derniers textes de Poulain soulignent bien cet aspect. Reste donc à traiter la question de l'utilité.

Le second argument de Poulain pour justifier une éducation des femmes identique à celle des hommes mobilise le concept de bon usage: les femmes sont aussi susceptibles que les hommes d'un bon usage de l'étude.

C'est donc une erreur populaire que de s'imaginer que l'étude est inutile aux femmes, parce dit-on qu'elles n'ont point de part aux emplois, pour lesquels on s'y applique. Elle leur est aussi nécessaire que le bonheur et la vertu; puisque sans cela on ne peut posséder parfaitement ni l'un ni l'autre. Elle l'est pour acquérir la justesse dans les pensées et la justice dans les actions : elle l'est pour nous bien connaitre nous-mêmes et les choses qui nous environnent, pour en faire un usage légitime, et pour régler nos passions, en modérant nos désirs. Se rendre habile pour entrer dans les charges et les

24. C'est déjà l'enjeu de l'Apologie de la science des dames, op. cit., p. 85 et suiv.

25. ES, I, p. 68-70.

26. Nous retrouvons ici B. Magné, «Éducation des femmes et féminisme chez Poullain de la Barre (1647-1723) », Colloque de Marseille. Le XVIIe siècle et l'éducation, Supplément au ${ }^{\circ} 88$ de la Revue Marseille, (1972), p. 117-127, p. 122. 
dignités c'est un des usages de la science : et il en faut acquérir le plus qu'on peut pour être Juge, ou Évêque, parce qu'on ne peut autrement se bien acquitter des fonctions de ces États ; mais non pas précisément pour y arriver et pour devenir plus heureux par la possession des honneurs et des avantages qu'ils produisent, ce serait faire de la science un usage bas et sordide. (ES, II, p. 115)

[Les femmes] sont capables [de] faire [de l'étude] aussi un très bon usage, et d'en tirer les deux avantages que l'on en peut espérer, l'un d'avoir les connaissances claires et distinctes, que nous désirons naturellement, et dont le désir est souvent étouffé et anéanti par la confusion des pensées et par les besoins et les agitations de la vie ; et l'autre d'employer ces connaissances pour leur conduite particulière, et pour celle des autres dans les différents états de la société, dont on fait partie. (ES, II, p. 116).

Le tout n'est pas ici de déterminer qui peut savoir, mais de se demander, si l'on peut savoir, à quoi bon le faire. Par la notion de bon usage, c'est un triple intérêt que Poulain de la Barre confère à la science acquise par les dames : un intérêt pour chaque femme prise individuellement, un intérêt pour toutes les femmes et un intérêt pour la société prise dans son ensemble.

\section{(a) Le triple intérêt d'un bon usage de l'étude}

À l'échelle individuelle, l'argumentation de Poulain de la Barre repose sur l'idée que la connaissance est le moyen nécessaire pour atteindre le bonheur, la vertu ${ }^{27}$ et l'action droite. Un avare qui possède de nombreuses richesses et se connaît les posséder, dit Poulain, sera heureux du fait de cette connaissance (ES, II, p. 111). Cela dit, son bonheur sera sujet à une certaine instabilité - qu'il perde sa richesse, ou qu'il change d'avis sur la quantité d'argent nécessaire pour être riche, et son bonheur s'estompera immédiatement. La connaissance la plus propice au bonheur sera indépendante de la possession ou non de biens dont l'acquisition ne dépend pas entièrement de nous : il s'agira de «la science qu'on a de soi-même, et de toutes celles qui en dépendent » (ES, II, p. 111). Il en est de même pour la vertu et l'action droite: non seulement parce que Poulain identifie le bonheur et la vertu ( $\ll$ il suffi[t] pour estimer un homme heureux, de voir qu'il pratique la vertu», ES, II, p. 112) et qu'il affirme qu'un homme « ne peut lui-même s'estimer solidement heureux, sans s'apercevoir qu'il fait le bien » $(E S, \mathrm{II}, \mathrm{p} .112)$, mais encore parce que la science est tout autant nécessaire pour trouver le bonheur que pour le pratiquer - «C'est la persuasion qui fait agir » (ES, II, p. 112). À titre individuel, pour chaque être humain, et plus particulièrement pour chaque femme, le bon usage du savoir sera la condition sine qua non de l'accession au bonheur et de la pratique vertueuse. On ne saurait donc réduire l'étude, pour les femmes, à un simple

27. Sur les deux thèmes du bonheur et de la vertu dans leur rapport avec la connaissance, voir en particulier ES, II, p. 111-112. 
passe-temps $^{28}$, ni envisager qu'on les instruise de minces rudiments de science, par opposition au savoir plus complet dispensé aux hommes.

À l'échelle du «beau sexe» en général, l'éducation permettra de corriger les inégalités entre les hommes et les femmes qui ne dérivent pas de leur différence naturelle, mais sont le fruit de la coutume, des préjugés et d'intérêts mal compris. Ainsi Poulain écrit-il :

Cette petite discussion des plus signalés défauts, qu'on croit particuliers et naturels au beau Sexe, fait voir deux choses, l'une, qu'ils ne sont pas si considérables que le vulgaire se l'imagine ; et l'autre qu'ils peuvent être rejetés sur le peu d'éducation qu'on donne aux femmes, et que tels qu'ils soient, ils peuvent être corrigés par l'instruction dont elles ne sont pas moins capables que nous. (ES, II, p. 141)

L'infériorité dans laquelle les femmes sont placées est sociale, et non naturelle, et peut à ce titre être corrigée. Ce sera, à l'égard des femmes considérées toutes ensemble, la tâche de leur éducation.

Enfin, notre texte acquiert sa part la plus révolutionnaire dans la considération du bon usage de l'étude relativement aux emplois ${ }^{29}$ : femmes comme hommes sont susceptibles des mêmes charges dans la société - la réussite dans celles-ci ne nécessitant que l'étude et «[le] discernement et [la] justesse » (ES, II, p. 116). Les femmes ne sont pas naturellement incapables des charges traditionnellement masculines (y compris la direction d'un champ de bataille, ou l'office religieux): la même instruction que les hommes les rendrait aptes aux mêmes emplois. Mais dire qu'elles sont susceptibles du même bon usage du savoir que les hommes ne suffit pas à ce que le processus d'attribution des postes évolue. Se développent donc, dans un registre discursif différent, un appel de Poulain de la Barre à ce que les « emplois et [...] la grandeur du monde» $(E H$, I, p. 320) soient attribuées selon le mérite, et non selon «la naissance, le bien et la fortune » (ibid.), ainsi qu'une critique de l'ordre social existant. C'est, par le biais de l'obtention des emplois, toute l'organisation sociale qui est pensée à nouveaux frais. La société, comme corps, gagnerait à ce que ses fonctions capitales soient détenues par ceux et celles de ses membres qui sont les

28. On cerne ici la différence entre l'argumentation poulainienne et la majeure partie des défenses de l'éducation des dames qui l'ont précédée, dans le premier XVII ${ }^{\mathrm{e}}$ siècle. Linda Timmermans, dans L'Accès des femmes à la culture (1598-1715) (Paris, Honoré Champion, 1993, p. 291-292), rappelait par exemple que Jacques du Bosc, ne s'adressant qu'à des femmes de l'élite sociale qui n'avaient ni à s'occuper de leurs enfants (disposant de nourrices) ni de l'entretien du ménage (disposant de servantes), considérait que leur principal ennemi était l'ennui. Le bénéfice que l'on doit attendre à titre individuel de l'étude n'est donc ni la vertu, ni le bonheur, ni l'action droite, mais simplement l'occupation d'esprit.

29. Poulain se démarque ici d'Anna Maria van Schurman, qui défendait l'étude pour les femmes tout en affirmant leur devoir de se tenir à l'écart des charges publiques (Question célèbre. S'il est nécessaire, ou non, que les Filles soient sçavantes, trad. G. Colletet, Paris, Rolet le Duc, passim, mais par exemple : p. 51-52). 
plus compétent(e)s. En ce sens, l'instruction des dames permettrait un choix plus large en ajoutant des prétendantes aux prétendants existants, et l'on comprend ainsi que l'utilité de l'instruction mentionnée par StasimaquePoulain, dont nous nous efforçons d'épuiser le sens, acquiert ici une dimension sociale. Mais il faut bien souligner que la considération sur « le bien de la société » $(E H, \mathrm{I}, \mathrm{p} .325)$ est d'un ordre différent des réflexions sur l'égale capacité des hommes et des femmes aux emplois, qu'il n'y a nulle inférence directe de l'un à l'autre ${ }^{30}$. La société fonctionnera d'autant mieux que les charges seront attribuées selon le mérite, et en même temps femmes et hommes sont susceptibles d'un même bon usage de l'étude, par lequel ils mettront celle-ci au service des autres.

Mais Poulain de la Barre prête également attention aux changements qu'implique l'éducation accrue des dames sur le savoir et la pratique de la science. Autrement dit, affirmer que les femmes aussi peuvent et doivent savoir a pour conséquence de redéfinir le savoir en question ${ }^{31}$. Poulain adresse une critique forte et d'inspiration cartésienne à l'encontre des institutions du savoir existantes, qui reposent sur l'autorité des professeurs (le «symbole Académique », ED, II, p. 194), la pratique des langues anciennes et de la disputatio. À l'inverse, l'éducation des dames promouvra l'avènement d'un savoir nouveau, du fait précisément de leur exclusion jusqu'alors des institutions savantes: l'autorité sera remplacée par le dialogue entre des esprits raisonnables recherchant la vérité (« toute notre autorité naturelle se réduit au pouvoir de la raison », $E H, \mathrm{I}$, p. 309), les langues anciennes ne seront plus nécessaires et à la dispute succèdera la douceur. On comprend, par la négative, ce qu'est le mauvais usage de l'étude, commun aux " Prêtres des Égyptiens » (ES, I, p. 68) et aux maîtres des Écoles, et qui caractérise les institutions du savoir. La connaissance n'y est pas utilisée pour le bonheur et la vertu, le progrès vers l'égalité ni l'exercice d'une charge publique. Elle est employée au contraire dans un objectif de domination, lorsque le savant attire à soi « la créance des personnes simples et crédules » $(E S, \mathrm{I}, \mathrm{p} .76)$, corrompant ainsi « en tyrannie l'autorité » (EH, Préface, p. 314) qu'il a acquise.

Reste une difficulté dans la détermination du bon usage de l'étude : il arrive que Poulain n'envisage plus la connaissance comme le moyen pour atteindre le bonheur, mais qu'il identifie le second à la première. $\mathrm{Si}$ «[1]e bonheur consiste dans la connaissance » $(E S$, II, p. 111), s'il en est de même pour la vertu (ES, II, p. 112), alors la notion même d'usage devient problématique, ainsi que la possibilité qu'il soit bon ou mauvais. Il faut donc dire que de la même manière que le concept de droit posait problème dans le premier argument de Poulain, celui de bon usage ne va pas ici sans difficulté. Cela étant, il permet de prendre acte de trois décalages. Le premier

30. Remarquons ainsi que Poulain de la Barre ne se prononce pas en faveur de la stricte parité (EH, I, p. 325).

31. Cf. M.-F. Pellegrin, «La science parfaite. Savants et savantes chez Poulain de la Barre », Revue philosophique de la France et de l'étranger, 138, (2013), p. 377-392, p. 391. 
est entre la théorie et la pratique, en ce sens que l'accès à des connaissances n'implique pas nécessairement une action en conséquence, et qu'il faut distinguer la généralité du savoir et la particularité de l'occasion de l'appliquer. Le second sépare l'étude de la vie active: l'étude n'a pas chez Poulain de la Barre une visée autotélique, elle n'a aucune raison d'autonomie, mais est tournée vers son usage dans la vie quotidienne. Enfin, le dernier point tient à l'écart entre les niveaux individuel et collectif: le concept de bon usage du savoir prend tout son sens dans la réflexion sur les charges et les emplois, où il s'agit de mettre au service du public les connaissances que l'on a acquises.

\section{(b) L'amour de l'étude selon Émilie du Châtelet}

Si le principal usage de l'étude tient dans son application aux charges de la société, on sait cependant que les «femmes savantes» des XVII ${ }^{\mathrm{e}}$ et XVIII ${ }^{\mathrm{e}}$ siècles n'ont pas occupé des fonctions dans lesquelles elles eussent été à même d'employer leur savoir. Comment maintenir l'appel à l'éducation des femmes lorsque celle-ci est coupée de cette finalité ? C'est dans cette tension que se déploie la pensée d'Émilie du Châtelet. Ses réflexions sur l'éducation des dames peuvent être comprises comme une interrogation du concept poulainien de «bon usage» de l'étude, en ce que la marquise est intimement consciente de la déconnexion, pour les femmes, des études et de l'accession aux emplois à « responsabilité ». Elle propose ainsi une argumentation féministe en faveur de l'éducation qui prend acte de ce que les femmes ne peuvent pas faire un «bon usage» public de l'étude. Son Discours sur le bonheur (rédigé entre 1746 et 1747, mais publié de manière posthume en 1779) interroge ainsi le rôle que doit jouer l'étude, dans ce contexte $^{32}$, pour le bonheur des dames, tout en soutenant que le changement de son état n'est pas ce que l'on doit désirer en vue du bonheur.

Si celui-ci n'y est pas défini de manière unifiée, le texte d'Émilie du Châtelet est gouverné par l'idée que le bonheur sera d'autant plus facile à atteindre qu'il tiendra dans des sentiments agréables dont l'apparition dépendra plus de nous-même que d'autrui. C'est par exemple au nom de ce critère qu'elle présentera ses réserves à l'encontre de la passion de l'ambition. Cette dernière, relativement au bonheur, est source de plus d'embarras que de succès : non pas " parce que l'ambition désire toujours, car c'est assurément un grand bien, mais c'est parce que de toutes les passions c'est celle qui met le plus notre bonheur dans la dépendance des autres $»^{33}$. Dans le cadre de l'examen du bonheur féminin, Émilie

32. Sur la critique de l'exclusion des femmes hors de la société, et pour une mise en relation du Discours avec les textes physiques de la marquise, on se reportera à R. Hagengruber, « If I Were King ! Morals and Physics in Emilie Du Châtelet's Subtle Thoughts on Liberty », in Women and Liberty, 1600-1800 : Philosophical Essays, J. Broad et K. Detlefsen (éd.), New York, OUP, 2017, p. 195-205.

33. Émilie du Châtelet, Discours sur le bonheur, Paris, Payot \& Rivages, 1997, p. 52. 
du Châtelet est amenée à accorder une attention particulière à l'amour de l'étude ${ }^{34}$. Moins notre bonheur dépend des autres, plus il nous est aisé d'être heureux:

Par cette raison d'indépendance, l'amour de l'étude est de toutes les passions celle qui contribue le plus à notre bonheur. Dans l'amour de l'étude se trouve renfermée une passion dont une âme élevée n'est jamais entièrement exempte, celle de la gloire ; il n'y a même que cette manière d'en acquérir pour la moitié du monde, et c'est cette moitié justement à qui l'éducation en ôte les moyens, et en rend le goût impossible. Il est certain que l'amour de l'étude est bien moins nécessaire au bonheur des hommes qu'à celui des femmes. Les hommes ont une infinité de ressources pour être heureux, qui manquent entièrement aux femmes. Ils ont bien d'autres moyens d'arriver à la gloire. (Discours sur le bonheur, op. cit., p. 52-53)

Ce texte est crucial : il avance plusieurs thèses dont certaines prennent le contrepied de l'argumentation de Poulain. L'amour de l'étude apparaît d'abord comme la passion la plus à même de nous faire accéder au bonheur, puisque les sentiments agréables qui en découlent dépendent peu d'autrui. Cela étant, Émilie du Châtelet introduit d'emblée une nuance capitale: l'amour de l'étude est moins nécessaire au bonheur des hommes qu'à celui des femmes. L'étude est en effet le seul moyen dont ces dernières disposent pour satisfaire une passion qui leur est commune avec les hommes, à savoir la gloire, le désir de se faire un nom ou de laisser une trace. C'est l'une des passions les plus communément partagées, mais alors qu'elle peut être source de bonheur, car satisfaite, chez les hommes, par le biais de la carrière qui permet de « rendre ses talents utiles à son pays et de servir ses concitoyens, soit par son habileté pour l'art de la guerre, ou par ses talents pour le gouvernement, ou les négociations $»^{35}$, « il ne [reste à la femme] que l'étude pour la consoler de toutes les exclusions et de toutes les dépendances auxquelles elle se trouve condamnée par l'état ${ }^{36}$. Les carrières administratives, juridiques, politiques, militaires ou de grand négoce étant fermées aux femmes, celles-ci n'ont la possibilité d'exceller que dans le seul domaine des études. Et alors que plaisir et excellence dans l'étude impliquent, pour les hommes, d'obtenir la responsabilité de charges glorieuses, elles n'aboutissent à aucun débouché social pour les femmes.

La différence entre les arguments d'Émilie du Châtelet et Poulain de la Barre se situe bien à l'endroit du second concept médian entre l'égalité des esprits et la nécessité de l'étude : le bon usage du savoir. Poulain prétend certes que ce serait faire mauvais usage du savoir que de l'utiliser dans le cadre d'un emploi en vue de la gloire que l'on pourrait retirer de cette

34. Voir V. Le Ru, Émilie du Châtelet philosophe, Paris, Classiques Garnier, 2019 (en particulier : p. 66 et suiv.).

35. Discours sur le bonheur, op. cit., p. 53.

36. Ib. 
position. On ne trouve pas la même condamnation de la gloire dans l'œuvre d'Émilie du Châtelet. Mais le point important tient à ce que Poulain de la Barre affirme que le bon usage du savoir est tourné vers autrui : c'est la mise au service de l'autre, par le biais des charges et des emplois, de ses compétences. La marquise du Châtelet, au contraire, prend acte de la séparation pour les femmes entre l'entreprise de savoir, d'un côté, et les charges publiques, de l'autre. Elle souhaite bien sûr que les femmes se voient ouvrir l'accès aux savoirs réservés aux hommes (c'est un thème présent dès la Préface de sa traduction de la Fable des abeilles ${ }^{37}$ ); elle considère en outre que l'exclusion des femmes hors des charges importantes est une caractéristique de leur état, plutôt qu'une conséquence de leur nature. Mais Émilie du Châtelet elle-même est l'illustration de ce qu'une femme, bien qu'éduquée comme les hommes, n'a pas l'occasion d'exercer les fonctions qui correspondent à ses connaissances. Ceci remet en cause jusqu'à la plénitude du bonheur atteint par les femmes dans l'étude. Alors que par l'amour de celle-ci, il s'agissait de s'affranchir de toute dépendance à l'égard d'autrui, l'étude peut être recherchée par les femmes comme seule voie d'accès à la gloire, et ainsi retomber sous une raison de dépendance. D'un point de vue descriptif, enfin, c'est le concept même de bon usage de l'étude qui devient genré : pour les hommes, il consistera bien dans l'exercice de fonctions adéquates aux connaissances acquises, alors que la notion d'usage perdra de sa pertinence pour les femmes.

\section{Conclusion}

Démontrer une capacité de l'esprit n'implique pas d'avoir démontré un droit, un usage effectif ni une utilité. L'argumentation poulainienne propose deux concepts jouant le rôle de médiation : celui d'un droit sur la vérité et celui d'un bon usage de l'étude. En faisant le pont entre théorie de la connaissance et philosophie politique, ils permettent de répondre à un certain nombre d'arguments misogynes qui s'efforcent de déconnecter les capacités intellectuelles des femmes de la nécessité de leur éducation.

Mais ces concepts recouvrent des apories - un droit sur une réalité imprescriptible; l'identité de la connaissance, du bonheur et de la vertu difficilement compatible avec l'idée d'un bon/mauvais usage de l'étude -, qui trouvent toutes deux leur racine dans la bonté de la science. La première tient en effet à l'inadéquation de ces biens particuliers que sont la science et la vérité par rapport au droit des biens. La seconde repose quant à elle sur une dualité au cour de la connaissance, à la fois bien à rechercher pour soi et moyen vers des fins qui la dépassent. La philosophie d'Émilie du Châtelet

37. Voir E. Muceni, " "De la mauvaise éducation des filles". L'adaptation de La Fable des abeilles par Émilie du Châtelet », La Lettre clandestine, 27, (2019), p. 215-237. 
nous est apparue traversée de cette même dernière tension - elle qui saisit l'étude par sa raison d'indépendance et comme moyen de satisfaire la passion de gloire.

Demander «qui peut savoir?», au croisement de l'épistémologie et de la pensée politique, c'est bien déployer sa pensée au cœur de cette ambivalence. 